\title{
3
}

\section{EVOLUTIONARY METHODS IN DESIGN: DISCUSSION}

\author{
MIHALY LENART \\ University of Kassel, Germany \\ AND \\ MARY LOU MAHER \\ University of Sydney, Australia
}

There are numerous approaches to modeling or describing the design process by using formal methods. Different approaches are taken for tackling different tasks or for modeling different aspects of the design process. For example, formal grammars are used for capturing generative tasks or knowledge-based and case-based reasoning for utilizing domain knowledge or expertise in order to find solutions in a large and complex search space. In recent years evolutionary methods became popular in engineering and computer science for solving hard, and with other methods sometimes intractable problems. They have proven to be successful in such areas where there is little domain knowledge available and the solution needs not to be perfect, just good enough for the particular task.

Research on evolutionary methods started in the 1950s and the first computational models were developed in the 1960 s by I. Rechenberg, H. P. Schwefel and L. Fogel. A significant development came with J. Holland's and K. De Jong's work on Genetic Algorithms (GAs) in the 1970s. Since the early 1980s there has been an explosion of research and the number of new applications in science and engineering has been growing exponentially. In design, evolutionary methods have first been applied to optimization problems. More recently, they are also used for solving design problems which require creativity. The main concern of the papers presented at this workshop and the subsequent discussion was the role of evolutionary methods in design exploration, in particular, how evolutionary methods can support human creativity in the design process.

\section{Papers}

This session included two paper presentations:

1. Modelling Design Exploration as Co-Evolution: A Combined Gene Approach by Mary Lou Maher, Josiah Poon and Sylvie Boulanger. 


\section{Evolving Building Blocks for Design Using Genetic Engineering: A Formal} Approach by John S. Gero and Vladimir A. Kazakov.

The presentations showed two different approaches to the use of GAs in formalizing aspects of the design process. The first presentation was concerned with how GAs provide a mechanism for change over time and applied this mechanism in a way that allowed the design focus to change as well as the design solution. This was modeled as a co-evolutionary process in which two spaces co-evolve: the problem definition space and the solution space. The presentation emphasized the focus on exploration rather than on search that is characteristic for other computer based design models. The co-evolutionary paradigm has been chosen for it is less deterministic than traditional evolutionary paradigms. Problem solutions and definitions cooperate in order to improve their fitness. Fitness is defined, however, only locally which means that the fitness function, i.e., the focus of the design process might change as new generations evolve.

The second presentation was concerned with how GAs provide a basis for identifying representations that achieve a high level of performance. This mechanism is applied in a manner that is analogous to genetic engineering in which the representation of design genes evolved. The approach allowed the representation of design knowledge to change in response to good performance. It generates building blocks of a design language, starting from simple blocks from which successively more and more complex ones will be generated during the design process. Analogous to genetic engineering, emerging patterns are recognized and used for subsequent evaluation. The evaluation also identifies bad genes in which case either the proliferation of bad genes is cut off by halting the generation process or the bad genes are "treated" in order to get rid of undesirable features.

\section{Discussion}

The issues raised during the discussion were directed at one or the other presentation and are presented here according to the presentation that raised the particular issue.

\subsection{CO-EVOLUTION ISSUES}

Three major issues were raised:

\section{Should the problem definition change or should it be refined during} co-evolution?

This discussion raises a basic question of whether formal methods developed for solving a particular design problem should be allowed to change the design problem in the course of the process or should the formal methods be refining and adding to the problem definition. In other words, the question is how to define design exploration; where are the limits for changing the problem 
definition and at which abstraction (meta) level we want to carry out such changes.

The discussion introduced a few scenarios in which the problem definition would be changed and whether the problem should have been restated at a higher abstraction level rather than changed at a lower level. In particular, the design of a bridge was discussed where the initial design may prove to be intractable and the designer may change his mind and choose to design a tunnel crossing. This, as an example of a change in problem definition, could also be considered a refinement of a problem definition of designing a harbor crossing.

\section{Do the dominant criteria eliminate consideration of other criteria?}

This discussion raises the issue of loss of information when using GAs. When allowing the problem definition as a set of criteria to evolve according to a fitness function, it is possible that some initial criteria may be lost due to poor performance. In the model of design exploration as co-evolution, this potential loss may mean that the formal method does not solve the initial problem at all but solves some evolved representation of the problem. This problem is related to the lack of global fitness and thus to the possibility of changing focus in the course of the design process. There was some concern as to whether this was an appropriate model for CAD.

3. Do such formal methods need to model a global set of design criteria, or is it reasonable to model the fitness as a set of local criteria?

This discussion dominated the discussion period by raising an issue that could not be resolved. The model of co-evolution that uses the combined gene approach relies on a local fitness function that is defined within a genotype. This local fitness function provides the criteria for evaluating the individual genotype and there is no model of a global fitness. The global fitness introduced in the paper was used to track progress but was not used to guide the evolutionary process.

The arguments for a global fitness include: a set of criteria by which all design solutions are evaluated means that there is a common unit of measure for fitness; global criteria means that there is a representation of the problem being solved that is related to the context rather than the solution; a global fitness can directly be compared to the original problem as defined by the user's initial requirements. The lack of global fitness means also that the process does not converge and after a certain number of steps there is no way to tell what we have accomplished so far and where to stop.

The arguments for a local fitness include: each design solution should be evaluated on its own merit rather than on a predefined ruler; a local fitness allows other criteria to be introduced based on the solutions being considered; local criteria need not relate to the original problem definition since the initial re- 
quirements for a design problem may not be an appropriate characterisation of the need.

\subsection{GENETIC ENGINEERING ISSUES}

Three major issues were raised:

1. Is the correlation between good genes and performance causal or statistical? The concern in raising this issue is in the use of the genetic engineering approach to develop representations of design knowledge that are used as a basis for new designs. If the genes are evolved according to performance without an understanding how the performance relates to the genes in a causal way, then new representations are statistically good. A lack of causal understanding may cause the evolution to follow the limited measure of performance. The discussion was resolved that the presented method did rely on a statistical correlation and that this was as good a correlation as any other.

2. Are the evolved genes specialised for a specific criteria?

Given that the new genes evolved in response to performance of a specified criteria the implication is that these are specialised genes. This lead to a similar, related issue of whether the evolved genes could be used for other purposes. A flexibility of representation would be sacrificed because the method relies on a fixed performance measure.

\section{Can a bad gene be used again?}

This issue is similar to the loss of information issue raised in Section 2.1. If the bad genes are discouraged from being used, does this result in a loss of information in the genotype representation? The discussion brought out the details of what is meant by a bad gene. In fact, genotype representations are not modified, but certain combinations are encouraged and others are discouraged. This means that theoretically there is no loss of information. Practically there may be a loss if certain genotypes are discouraged to a point where they are not used at all. However, if the performance criteria changes, these bad genes may start to reappear again.

\section{Summary}

The discussion could be summarised as two statements and two questions:

Statement 1: The use of GAs presented and discussed in this session employs a mechanism to change the design space: the co-evolution methods change the representation of the design requirements and design solutions, and the genetic engineering method changes the representation of design knowledge.

Statement 2: Evolutionary methods provide a set of mechanisms for generating design solutions and rely on evaluation knowledge to guide the design process. 
Question 1: How do we know if the changing design space is still relevant? The co-evolution methods change the design requirements to a point where they may not have anything in common with the original requirements. The genetic engineering method changes the design knowledge to a point where the original design knowledge representation may never be used at all.

Question 2: Do these evolutionary methods do something fundamentally different to other formal methods of design? What kind of design problems can be tackled with evolutionary methods better than with other formal methods?

The answers to these two questions point to new research directions for formal design methods based on evolutionary approaches. 